\title{
The use of correlated stimulus information in card sorting'
}

\author{
JOHN MORTON 2 \\ APPLIED PSYCHOLOGY RESEARCH UNIT, CAMBRIDGE
}

Subjects were required to sort three packs of cards into six piles. On the cards of one pack were a number (one to six) of $x$ s. In the second pack the cards had a single digit (1 to 6). On the third pack the two kinds of information were perfectly correlated, i.e., there were three $3 s$, four $4 s$, etc. This pack was sorted more quickly than either of the other two. It is provisionally concluded that the Ss were processing information in parallel rather than serially, and that evidence from two sensory analyzers was being combined rather than the outcome from two decision mechanisms. This conclusion is tempered by the observation that only a detailed study of the reaction times to individual stimuli can provide us with an unqualified verdict.

The effects of correlated stimulus information upon performance vary with the nature of the task and the nature of the correlated dimensions in a fairly complex way. Sometimes the added dimension improves performance, sometimes there is no effect (see Garner, 1962; Garner \& Lee, 1962; Lockhead, 1966). The experiments hitherto performed on this topic have usually measured discriminability. In the present study the dependent variable is time. The two dimensions used were the number of objects on a card and the names of arabic numerals.

\section{EXPERIMENT 1 \\ Method}

Each of 18 paid college students sorted three packs of 36 cards 12 times. The packs each contained six examples of six different cards and were sorted into six piles corresponding to the numbers 1-6. The packs were termed (a) the Numerosity pack (N) on which there were 1 to $6 \mathrm{xs}$, (b) the Digit pack (D) on which one of the digits 1-6 was typed, and (c) the Mixed pack (M) on which these two aspects were perfectly correlated. On the N-pack the xs were typed on the cards in regular patterns, all the patterns being the same for all cases of a particular number. This was done in order to simplify the task of counting, and make it almost as easy as the recognition of an arabic numeral. Thus, in the M-pack there were six cards with one 1 , six with two 2 s, etc. The pattern of the digits in the M-pack was identical to that of the xs in the N-pack. In the M-pack, therefore, Ss could use either or both of the attributes in the sorting task. Individual Ss sorted the three packs in an order determined by a repeated modified Latin square design which was counterbalanced across Ss. The Ss could be regarded as having 12 trials during each of which all the packs were sorted once. Order within trials across Ss was thus balanced. Following the sixth trial, Ss had a 5-min rest. The Ss were instructed to "sort as quickly as possible without making any errors." The packs were shuffled by hand. The Ss held the cards face down and sorted them face upwards. Errors were rare and have been ignored.

\section{Results}

The data were subjected to an analysis of variance ignoring practice effects within trials. The two main effects were highly significant, that due to the differences between the packs yielding $\mathrm{F}=71.55, \mathrm{df}=2 / 34, \mathrm{p}<.001$, and that due to practice giving $F=260.95, \mathrm{df}=1 / 17, \mathrm{p}<.001$. There was also a significant interaction between packs and practice with

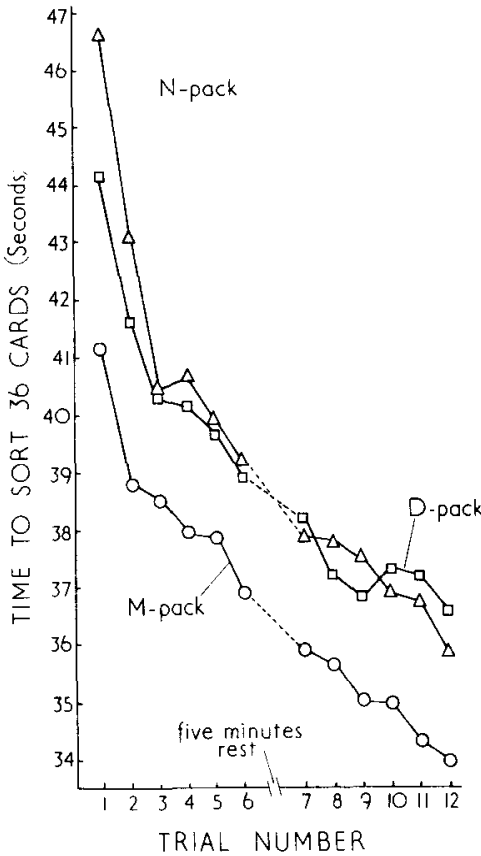

Fig. 1. The average time, over $18 \mathrm{Ss}$, to sort a pack of 36 cards on which there was either a single digit (D-pack), a number of $x s$ (N-pack), or both these kinds of information in a correlated form, such as five $5 \mathrm{~s}$ (M-pack).

$\mathrm{F}=5.88, \mathrm{df}=2 / 34, \mathrm{p}<.01$. The data are presented in Fig. 1. It is clear that there is a great advantage to the Ss of having the two attributes. For all Ss the time to sort the M-pack was significantly shorter than for either of the two other packs, the average difference being $2 \mathrm{sec}$. The interaction between packs and practice seems to be entirely due to the rapid improvement on the $\mathrm{N}$-pack on the first two trials relative to the other two packs. This could be because the Ss had to learn to recognize the patterns of the stimuli instead of having to count them.

\section{Discussion}

To discuss alternative explanations it is necessary to distinguish two stages of information processing each of which would take a variable amount of time. The first involves processing prior to a categorical outcome. The second is the production of the categorical outcome which could be either the implicit response of a digit name (which then would be used to mediate the sorting response) or the outcome of matching the card in the hand with that on one of the piles. From introspective evidence the latter method of performing the task seems unlikely to be a true account but this possibility does not affect the argument which follows.

\section{Serial Processing}

If the two sources of information on the M-cards could only be processed serially and independently prior to a categorical 
decision, it would be necessary to process only one dimension in order to produce the correct response. If the second dimension were also analyzed, the time taken for the M-pack would be longer than for the other packs. As the time for the M-pack was in fact less than for the other packs the Ss could not have processed both dimensions serially.

Serial-selective model. If only one dimension were analyzed the choice of which dimension to analyze could not be random, for if it were, performance on the M-pack would be equal to the average performance on the other two. Instead, the choice of dimension would have to be dictated by the state of the mechanisms which processed the two kinds of information, and that dimension chosen for analysis whose mechanisms seemed most likely to be more efficient for the next M-pack (or M-card: if the choice of dimension changes within a pack). To test this explanation the time for the M-pack on every trial was compared with the faster of the times on that trial for the other two packs, using the Wilcoxon test. The superiority of the M-pack was maintained over all 12 trials, significantly so for 10 of them (at $p<.01$ ). All 18 Ss were on average faster on the M-pack than on the better of the other two. The difference was significant for 13 of the $18 \mathrm{Ss}$. Thus, the serial-selective model will not hold if the dimension selected for analysis were the same for an entire M-pack. (It should be noted that this is a very strong test since no allowance was made for the fact that when comparing the best of two scores against a third score, random factors work against the latter score.)

It is of course still possible that the dimension selected for analysis changes during a single pack. In the limit, the relevant decision may be made prior to every individual card. It should be noted that the a priori implausibility of this refinement is reduced if we consider that the effect (that the dimension which will give the faster outcome on the next trial is selected) may be indirect. Thus, if the dimension chosen alternates from card to card, and if the analysis systems become more efficient when given time to recover from a previous analysis then we would have the condition required for the serial-selective model to produce the main result-an apparent facilitation. If some preselection of dimension were made, one would expect the difference between the M-pack and the other two to be less if one of the two sources of information were appreciably more difficult, since in such a case, the easier of the two would be selected on a majority of occasions, and performance on the two-dimension case would approach that on the faster of the individual cases. In the present experiment, such a situation was the case on the first two trials, where the average time for the D-pack was much less than that for the N-pack. The average difference between the M-pack and the other two was, however, as great or greater on the first two trials as on the rest. The prediction unfortunately assumes that the variance in reaction time to individual cards remains constant from trial to trial. If in the present experiment this variance were substantially greater on the first two trials than on the subsequent trials then the serial-selective model would still hold. The ultimate decision will require the measurement of reaction times to individual cards.

This model would similarly predict that for individual Ss, the greater the difference in performance between the $\mathrm{N}$ - and D-packs, the less the difference between the times for the M-pack and the better of the other two. This prediction was tested over the second half of the trials, by which time the difference between $A$ and D was relatively stable. A nonsignificant correlation was found between the two measures (rho $=.37$ ). Such a value allows one neither to accept nor reject the theory. In addition, without precise data on the variance, of the reaction times to individual cards of the different Ss, it is not possible to make a final assessment.

One might note that the data of Todd (reported in
Woodworth \& Schlosberg, 1954, p. 26) is readily amenable to account by an unmodified serial-selective model. He presented Ss with a light, an electric shock or a sound, either singly or in simultaneous combination. A simple reaction was required to any stimulus or stimulus combination. The time to react to the light was larger than to either of the other stimuli for all of the three Ss who were used. When the light stimulus was paired with either shock or sound the reaction time was no greater than that to the paired stimulus. When shock and sound were paired, or when all three stimuli were presented, all three Ss responded more quickly than to any of the individual stimuli. This result could, of course, equally be accounted for by a parallel model but serves to exemplify the kind of data which would satisfy the serial model.

Digit-redundancy model. One final possible serial model would assume that only the digit information is processed. The improvement in performance in the $\mathbf{M}$ condition would be accounted for by supposing that when a number of examples of a digit are present the recognition of the digit is facilitated. (This would represent the parallel processing of information within a single dimension, the term "dimension" being used in the original sense of this paper.) One Morton experiment (in press) indirectly relevant to this problem compared the time taken to sort the D-pack with the time to sort a pack in which a variable number ( 1 to 6 ) of the digits 1 to 6 occurred. There were 12 Ss who sorted each pack three times. The time for sorting the D-pack was significantly faster than that for the other pack $(p<.005)$. This result was explained by supposing there to be interference from the numerosity, which in this case is irrelevant. There may of course still be some gain in the recognition of the digits which is totally negated by the interference. However, it seems plausible to argue that if numerosity information affects behavior when it is irrelevant, then it must surely have some potency when it is relevant. Thus, although it is possible that there is some gain in digit recognition with the M-pack, it does not seem possible to attribute all the gain to this factor. The possibility of it having any effect was tested more directly in Experiment 2.

\section{EXPERIMENT 2 \\ Method}

Twelve Naval Ratings sorted two packs of cards 11 times. One pack was identical to the D-pack in Experiment 1, i.e., the cards had a single digit ( 1 to 6 ) on them. Each card in the second pack (the 3D-pack) had three identical digits. There were six examples of each digit, making a total of 36 cards in each pack. Six Ss sorted the D-pack first and six the 3D-pack.

\section{Results}

The mean time for the D-pack was $40.9 \mathrm{sec}$ and that for the 3D-pack was $40.7 \mathrm{sec}$. The difference between the mean times did not reach significance. An analysis of variance on the last eight trials showed a significant practice effect $(F=5.05$; $\mathrm{df}=7 / 77 ; \mathrm{p}<.001$ ). There was, however, no interaction between trials and packs, which might have been the case if the Ss had to learn to use the redundancy on the 3D cards. It is clear that there is no facilitation whatsoever arising from the presence of more than one digit in the card.

\section{Parallel Processing}

\section{Discussion}

For these reasons it is concluded that the Ss are more likely to have processed the information from the two sources in parallel. In the following discussion it is assumed that the sensory processes provide information relevant to a categorical decision over time, such decision being taken after the accumulation of a certain amount of information. There are two broad types of models consistent with the result.

Evidence-combination. Relevant information from the two 
sources converges at the decision point and adds. The decision criterion would be reached more rapidly with simultaneous processing of the two dimensions than with either alone. This formulation is equivalent to the suggested method of interaction of contextual and sensory information in word recognition in the Logogen Model (Morton, 1969).

Decision-combination. The categorization processes for digit recognition and numerosity could be independent. Given variation in the time to reach a decision for each of the dimensions, and given that the internal state leading to a sorting response is affected by the first available categorical outcome then it would follow that the M-pack would be sorted more rapidly. This would be equivalent to an inclusive-disjunctive model for interaction in experiments on detection or discrimination (e.g., Wickelgren, 1967).

The latter model makes the same prediction as the serial-selective model, in that the greater the difference in response time between the various dimensions, the less the expected gain with two dimensions together compared with the faster of the individual dimensions. It was shown above that this prediction was not upheld. The decision-combination model cannot be rejected completely, however. As with the serial-selective model (which in its extreme form is indistinguishable in its detailed predictions from the decision-combination model) the prediction about the relationship between the amount of gain with two dimensions and the differences between the dimensions is contingent on the variance of the individual decision times in the different conditions.

However, the difference between the M-pack and the other two remains approximately constant from the third trial onwards. For the model to be applicable, the variance in the decision times on both the $\mathrm{N}$ - and $\mathrm{D}$-packs would thus have to remain constant over these trials in spite of a decrease of about $10 \%$ in the overall time (which includes sorting time as well as decision time). In addition, assuming normal distributions, it can be shown that to obtain the observed result on the first trial the variance of the decision times on the N-pack on that trial would have to be, on average, twice that on subsequent trials. The plausibility of these requirements is difficult to assess and a study of reaction times to individual stimuli is necessary. From such a study the applicability of the decision-combination model can be assessed through a direct comparison of the distributions of the reaction times. It should be noted, however, that if one allows the possibility of correlation between the distributions of decision times on the two dimensions, as would be caused, for example, by fluctuations in general attention, predictions from any of the alternative models becomes less exact. From the evidencecombination model in its simple form we can make no prediction about the relationship between the expected gain and the differences between the dimensions. To do so we would have to specify in more detail the nature of the combination of evidence and the rates of evidence accumulation in the various conditions as well as considering any possible changes in decision criteria.

The simple forms of either a decision-combination model, or, as indicated, a serial-selective model could account for the data of Todd, referred to above. Whether or not this is due to the fact that only a simple detection was required, or whether it is due to the different sources of information being on different sensory modalities is uncertain.

There are at least two other studies which are related. Corcoran (1967) studied the speed of classification of stimulus cards varying in a binary fashion in area and brightness. His conclusions were that his Ss initially classified the two dimensions serially. After practice, however, they were able to process the dimensions in parallel. His data do not permit a distinction to be made between decision-combination and evidence-combination. Equally, the classification of serial and parallel models made by Egeth (1966) is not strictly relevant to the present study, as the types of models and the predictions from them were specific to his task. In his experiment the Ss had to respond "same" or "different" to a pair of visual forms which were identical or differed on from one to three dimensions, some of which may have been irrelevant. The time taken to respond was measured. Egeth concludes that in his task the Ss processed the dimensions serially.

Any lack of agreement between the conclusions of these studies and the present one merely reflects the differences in the nature of the task and of the stimuli in the various experiments. Any complete picture of the organism will probably have to allow for both methods of information processing. In addition, any final decision about the mode of processing in any particular task will require a more detailed examination of the data than is possible with the current technique.

CORCORAN, D. W. J. Serial and parallel classification. British Journal of Psychology, 1967, 58, 197-203.

EGETH, H. E. Parallel versus serial processes in multidimensional stimulus discrimination. Perception \& Psychophysics, 1967, 1, 245-252.

GARNER, W. R. Uncertainty and structure as psychological concepts. New York: Wiley, 1962.

GARNER, W. R., \& LEE, W. An analysis of redundancy in perceptual discrimination. Perceptual \& Motor Skills, 1962, 15, 367-388.

LOCKHEAD, G. R. Effects of dimensional redundancy on visual discrimination. Journal of Experimental Psychology, 1966, 72, 95-104.

MORTON, J. Categories of interference: Verbal mediation and conflict in card sorting. British Journal of Psychology, in press.

MORTON, J. The interaction of information in word recognition. Psychological Review, 1969, 76.

WICKELGREN, W. A. Strength theories of disjunctive visual detection. Perception \& Psychophysics, 1967, 8, 331-337.

WOODWORTH, R. S., \& SCHLOSBERG, A. Experimental psychology. New York: Holt, 1954.

\section{NOTES}

1. Experiment 1 was performed and work on the paper begun while the author was Research Associate and Lecturer at Yale University, supported by Grant MH 14229 from the National Institute of Mental Health to Yale University. The assistance of Mrs. Kathleen A. Catanese is gratefully acknowledged. The study has benefited from discussions with Professor W. R. Garner.

2. Address: Medical Research Council, Applied Psychology Research Unit, 15 Chaucer Road, Cambridge, England.

(Accepted for publication January 6, 1969.) 